\title{
High Density Arrays of Micromirrors
}

\author{
J. A. Folta \\ J.Y. Decker \\ J. Kolman \\ C. Lee \\ J.M. Brase
}

February 1999

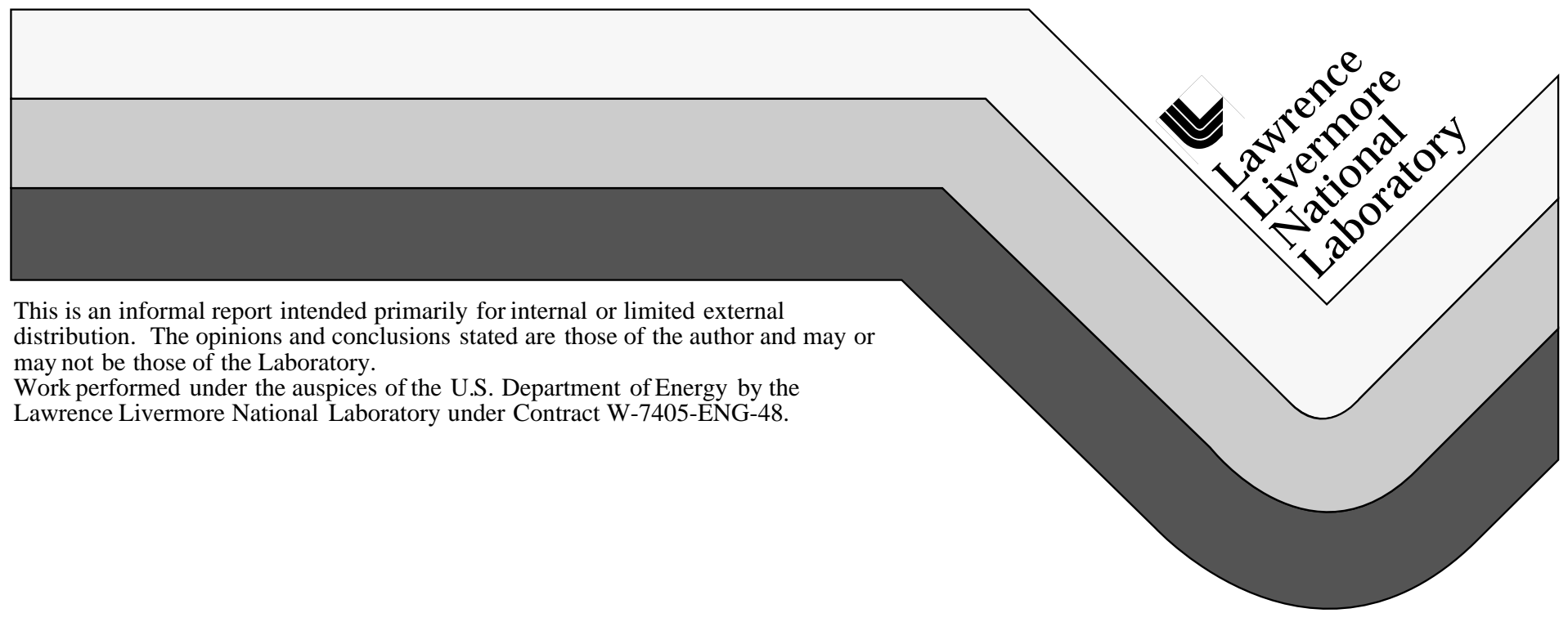




\section{DISCLAIMER}

This document was prepared as an account of work sponsored by an agency of the United States Government. Neither the United States Government nor the University of California nor any of their employees, makes any warranty, express or implied, or assumes any legal liability or responsibility for the accuracy, completeness, or usefulness of any information, apparatus, product, or process disclosed, or represents that its use would not infringe privately owned rights. Reference herein to any specific commercial product, process, or service by trade name, trademark, manufacturer, or otherwise, does not necessarily constitute or imply its endorsement, recommendation, or favoring by the United States Government or the University of California. The views and opinions of authors expressed herein do not necessarily state or reflect those of the United States Government or the University of California, and shall not be used for advertising or product endorsement purposes.

This report has been reproduced directly from the best available copy.

Available to DOE and DOE contractors from the Office of Scientific and Technical Information P.O. Box 62, Oak Ridge, TN 37831

Prices available from (423) 576-8401

Available to the public from the National Technical Information Service

U.S. Department of Commerce 5285 Port Royal Rd., Springfield, VA 22161 


\section{High Density Arrays of Micromirrors}

LDRD final report

2/23/99

Tracking code: 97-ERD-078

UCRL-ID-133164

Principal Investigator: James A. Folta

Final report authors: J. A. Folta, J. Y. Decker, J. Kolman, C. Lee, J. M. Brase

\section{Introduction}

We have been exploring designs for high-spatial-density micromirror arrays (MMA's) for use in maskless lithography, pattern generation of photolithographic masks, and adaptive optics (AO) for imaging and laser-beam control. In particular, MMAs offer a potentially high-payoff application to maskless lithography.

Lithography is the enabling technology for the $\$ 300 \mathrm{~B}$ worldwide semiconductor electronics industry, which in turn has been the underpinning for U.S. dominance in military technology. Semiconductor lithography at its essence is a technique for accurately and cost-effectively transferring binary design information from the masks onto silicon wafers. In this process the mask is nothing more than the physical carrier of that binary design information. Over the past three decades semiconductor electronics has been well served by optical lithography, which has provided a fourfold increase in device performance every three years (Moore's Law"). Recently, however, the technical demands on optical lithography required to maintain this exponential pace of performance growth has pushed lithographic technology to its limits. In particular, mask technology - the science of fabricating, inspecting, repairing, and protecting the binary master-pattern masks used to synthesize advanced integrated circuits level by level - is fast becoming the Achilles heel of advanced lithography systems.

In our proposed maskless lithography system, the "hard copy" masks are replaced by programmable arrays of binary (on/off) light valves or switches, which can be rewritten to assume any arbitrary pattern to mimic the mask patterns. The binary array can take on many forms and falls into a broad class of devices called Spatial Light Modulators (SLMs) capable of controlling phase and/or amplitude of light. The maskless lithography concept enjoys a number of attractive features:

1. The "physical carrier" of the master pattern in eliminated. The master pattern resides in computer memory in its purest form: information 
2. The master pattern is agile. Since it is resident in computer memory it can be easily upgraded or modified. Changes can be transmitted to all lithography systems in a factory and to all factories around the world, instantly.

3. Redundancy: the redundant pixel design is forgiving of single point defects so that patterns need not be defect-free This is a major cost saver.

4. No need for costly mask management outside the lithography system.

5. No physical transport of masks in/out of the stepper, and no scanning of masks. This significantly reduces the complexity and cost of stage hardware in stepper systems.

6. Conventional mask manufacturing technology (pattern writing, inspection, repair, substrate materials, etc.) is eliminated, removing the major impediment to the continued performance growth of lithography systems.

7. Implementation of the mask-free optical lithography concept would be a genuine revolution in lithography technology with major beneficial impact throughout the industry.

In order to demonstrate the feasibility of the maskless lithography concept, 3 technical developments are required.

1. SLM devices: Essential to the success of the maskless lithography concept is the development of a SLM device with sufficiently small pixels and sufficiently high capacity to carry the design information. Commercial SLM technologies lack either the requisite scalability to near-micron sizes or compatibility with UV sources.

2. Lithography system: In order to take full advantage of the freedom provided by the maskless lithography concept we must rethink the design of the entire lithography systems: sources, illuminations optics, imaging optics, stages, and alignment systems. Virtually all component specifications will be redefined. To accomplish this we need to develop the simulation tools for detailed end-to-end simulations of the lithography system which also include photoresist process simulations.

3. Electronic control and data transfer systems: Lithography in its purest forms is an information transfer process. In its classical embodiment information is transferred from a physical master pattern to a photoresist polymer on wafers using optical systems that transfer about $\sim 10^{10} \mathrm{bits} / \mathrm{sec}$. In the maskless lithography system the physical master pattern is replaced by a digital array which is controlled electronically and fed data at a rate commensurate with the optical transfer process. Such a control and high-speed transfer system must be designed and integrated with the MMA's. 


\section{Technical approaches}

A. MMA Design and analysis

The mirror elements are designed to be small enough so that many elements are required to print a minimum feature size on the wafer. This provides both a high degree of image placement accuracy and the redundancy makes the system forgiving of single-point failures in the array.

Mechanical, electrostatic, and microfabrication analysis tools are used to design MMA's.

Mechanical: Analysis: Mechanical analysis simulated deflection of the scaled-down mirrors in response to applied actuation forces. We used finite element modeling codes which already exist at LLNL and which have been successfully used to simulate a number of micro-electro-mechanical structures.

Electrostatic Analysis: Electrostatic analysis addressed scaling of the electrostatic forces used to deflect the mirror elements as well as examine crosstalk issues among adjacent mirrors. LLNL already had a modeling code for electrostatic actuation forces for micro-mechanical comb drives. It used a boundary element algorithm, and required fairly straightforward modifications for 3-D simulations of our MMA design.

Microfabrication process design: LLNL has extensive experience in the microfabrication of micro-electro-mechanical systems (MEMS) which provided a firm foundation on which to design novel micromirrors which are manufacturable. It was critical that proposed designs were limited to those which can be successfully fabricated. The design was also limited by 2 additional constraints: (1) The best approach to achieve the high data rates needed to rapidly reconfigure the mirror pattern was selected to be integrating the mirror array on top of an array of static random access memory (SRAM) devices. We limited our selection of materials and microfabrication processes to be compatible with integration with SRAM devices. (2) The final mirror surface must be extremely smooth in order to successfully deposit optical reflective multilayer coatings. One feasible approach is use of chemical mechanical polishing processes used to planarize IC's during the microfabrication process.

\section{B. Microfabrication}

As described below, we built test structures to verify their performance and to demonstrate feasibility of the high density arrays. 


\section{System Analysis}

We developed simulation codes required for a detailed end-to-end simulation of a maskless lithography system to analyze the expected performance of the full scale system. This anaysis will be used to set specifications on the MMAs and to guide development of a prototype maskless lithography system.

\section{Results and Discussions}

A. Micromirror development

a) Micromirror design

Our micromirror design was driven by the specifications required for the potentially high-payoff application of maskless lithography. The micromirror specifications included tiny ( $5 \mu \mathrm{m}$ or smaller) pixels capable of precise and small displacements $(\sim 90 \mathrm{~nm})$. Analysis of commercial SLM technologies (e.g. ferroelectric liquid crystals and hinged micro mirror designs) showed they lacked either the requisite scalability to near micron size or compatibility with UV sources.

In FY97 we selected a scaleable MMA design composed of highly compressible material sandwiched between two electrodes as depicted in Figure 1. (June, need to add the figure) Electrostatic forces compress the sandwiched layer when a voltage is applied between the two electrodes, moving the top (mirror) electrode. Direct integration of our MMA's onto SRAM devices provide the high speed addressing of the elements required for the targeted application areas. With proper configuration, our MMA can be used as either a phase modulator or an amplitude modulator. A laser beam reflected off the MMA will have its phase modulated spatially because of the varied path lengths. By letting a certain order of diffracted light pass through an imaging system, the phase modulation at the MMA surface can be converted into amplitude modulation at a desired imaging plane. Alternatively, amplitude modulation can be achieved by placing a static reflector near the mirrored actuators to create an array of Fabry-Perot (FP) cavities. Moving one mirror in a FP array will modulate the reflected amplitude. Increasing reflectivity of the FP mirrors results in higher finesse FP's requiring less piston displacement to achieve the same amplitude modulation. We expect these MMA's to be readily applied to many wavelengths including the ultraviolet (UV).

b) Electrostatic modeling

Electrostatic analysis of our design was done with a one-dimensional capacitive force model. This provided insight into the primary design parameters and estimates of equilibrium forces. Figure 2 illustrates the drive voltages required as a function of interlayer thickness and Young's modulus to provide a deflection of $90 \mathrm{~nm}$ (a quarter wavelength) needed for maskless lithography and mask pattern generation applications. For an interlayer material with a Young's Modulus of $0.005 \mathrm{MPa}$ 
(megaPascals) and a thickness of about $4 \mu \mathrm{m}$, we can get a deflection of $90 \mathrm{~nm}$ with moderate drive voltage of approximately $20 \mathrm{~V}$.

c) Mechanical modeling

Our finite element analysis provided insight into deflection uniformity and pixel crosstalk for various electrode configurations (including trenching, z-shaped bridges and serpentine interconnections). Mechanical modeling and analysis of the MMA was carried out with the implicit, nonlinear finite element code NIKE3D in order to identify materials and configurations that satisfy performance requirements for an electro-static deflection of $90 \mathrm{~nm}$. The deflection characteristics were studied through a representative problem of the deflection of the center mirror of a $3 \times 3$, nine mirror array. The main concern is to decouple mirrors so that motion of one mirror does not effect the position of its neighbors. A conductive z-shaped design for the top mirror/electrode was chosen as it allowed sufficient decoupling yet maintained electrical connections between mirrors.

d) Micromirror fabrication and evaluation

At the end of FY97, we began fabricating micromirror test structures for evaluation. Aerogels, which potentially satisfy the modulus requirement on the order of a few hundredths of $\mathrm{MPa}$, cannot be successfully and economically processed into the required thin films. Therefore, we investigated commercial elastomers as the compressible material for our device. However, at issue was whether elastomer films can be processed into uniform, thin ( $\sim \mu \mathrm{m}$ thick) films. Commercial elastomers are typically formulated for use as bulk samples or thick films (>70 $\mu \mathrm{m}$ thick).

In FY98, we successfully developed the fabrication process for producing thin $(\leq 4 \mu \mathrm{m})$, uniform elastomer films with commercial elastomers. We also developed a process for coating the elastomer films with thin $(\sim 50 \mathrm{~nm}) \mathrm{Au}$ films as the top electrode. We achieved surface roughness of less than $10 \mathrm{~nm} \mathrm{rms}$ for both the bare and coated elastomer films.

The second issue in using elastomers for MMAs was reduction of the elastomer bulk modulus. We identified a highly elastic material with a nearly negligible amount of hysteresis and with a measured bulk modulus of $0.5 \mathrm{MPa}$. Although extremely low for elastomers, the modulus is still an order of magnitude higher than desired. Prototype devices made with this elastomer produced lower displacements than required even for a $100-\mathrm{V}$ applied voltage. We carried out experiments for evaluating and reducing the bulk modulus of silicone elastomer films by patterning microvoids into the elastomer film. The microvoids reduce the effective stiffness of the films by allowing the elastomer to displace into the voids during compression. We developed a plasma-etch process for etching and patterning the elastomer films. At year end, we 
were evaluating the effectiveness of the microvoids at reducing the bulk moduli of these patterned films.

\section{B. Maskless lithography system analysis}

We first constructed a numerical model which describes a partially coherent imaging system such as commonly used in lithographic systems as well as a nonlinear photoresist. The imaging system is modeled by a bilinear transformation which accounts for both the condenser and projection optics. We developed algorithms which yield the output image of a lithography system given the input pattern (the "forward model"). The input signal can represent various types of static masks and spatial light modulators (i.e. our micromirror arrays). We also developed an algorithm for designing input signals for a lithography system with the goal of printing prescribed patterns onto semiconductor substrates (the "backward model"). Our algorithm attempts to work backwards, constructing an input signal which yields the desired printed pattern, by evaluating the quality of the pattern and iteratively adjusting the input. The forward model is used to do the evaluation, and a constrained conjugate gradients optimizer solves the iterative problem.

The backwards model is a signal processing challenge. We used an iterative optimization algorithm which minimized the error in the output. We use an estimate for the input pattern on the MMA, determine the resulting output image, and compare it to our desired output. After computing the error between the desired and actual outputs, we need a systematic way of adjusting the input pattern to yield a smaller error. This was done by computing the gradient vector (the set of derivatives of the error function with respect to each input pixel). This tells us whether a small increase in the reflectivity of a pixel will increase or decrease the error and by what amount. Knowing the gradient vector, we can simultaneously change the values of all pixels appropriately and decrease the error. This process is repeated until the error is acceptably small. We can compute the gradient in this problem exactly in a tractable amount of time. With slight modifications, we used an optimization algorithm which has proven to be successful, having generated excellent solutions to problems in tomography, synthetic aperture radar, crystallography, and other applications.

Recent developments have included techniques for greatly speeding up the required calculations and extensions of the model. The fast computational techniques allow practical sized problems to be solved in a reasonable amount or time, with no reduction in quality. The model extensions consist of the consideration of arbitrary complex objects and constraints which allow certain portions of the input signal to be fixed in value. This latter extension may be useful for modeling defects in multilayer 
masks. Another important avenue of future research is the development of an algorithm which produces a binary input signal.

\section{Micromirror designs for adaptive optics applications}

In FY98, strong programmatic pull drove us to investigate three new MMA designs which could be used as deformable mirrors for AO applications. Unlike maskless lithography where we require MMAs with tiny pixels ( few microns) and small displacements $(\sim 100 \mathrm{~nm})$, deformable mirrors for AO require MMAs with large pixels $(\sim 100 \mu \mathrm{m})$ ) and larger (up to $10 \mu \mathrm{m}$ ) displacement. No commercially available deformable mirrors meet LLNL's requirements. The three new micromirror designs were chosen for their potential to provide $10 \mu \mathrm{m}$ displacement over large arrays. All three offer operation at high spatial and temporal frequencies; proper design and coating of the micromirror surfaces would extend operation to high intensity illumination. The three designs are based on electrostatic, magnetic, and piezoelectric actuation. The three designs leverage LLNL's extensive capabilities in microfabrication and micro-electro-mechanical (MEM) devices. Our preliminary finite-element analysis of the actuation forces required for correcting a typical wavefront with high-spatial-frequency aberrations indicated that reasonable forces (tens of microNewtons) are required for actuating the individual mirror pixels. We are analyzing the feasibility of these new DM designs for AO applications in FY99.

\section{Summary}

We established and achieved our goal to (1) fabricate and evaluate test structures based on the micromirror design optimized for maskless lithography applications, (2) perform system analysis and code development for the maskless lithography concept, and (3) identify specifications for MMAs for LLNL's AO applications and conceptualize new devices. 
Figure 1: Conceptual drawing of micromirrror design

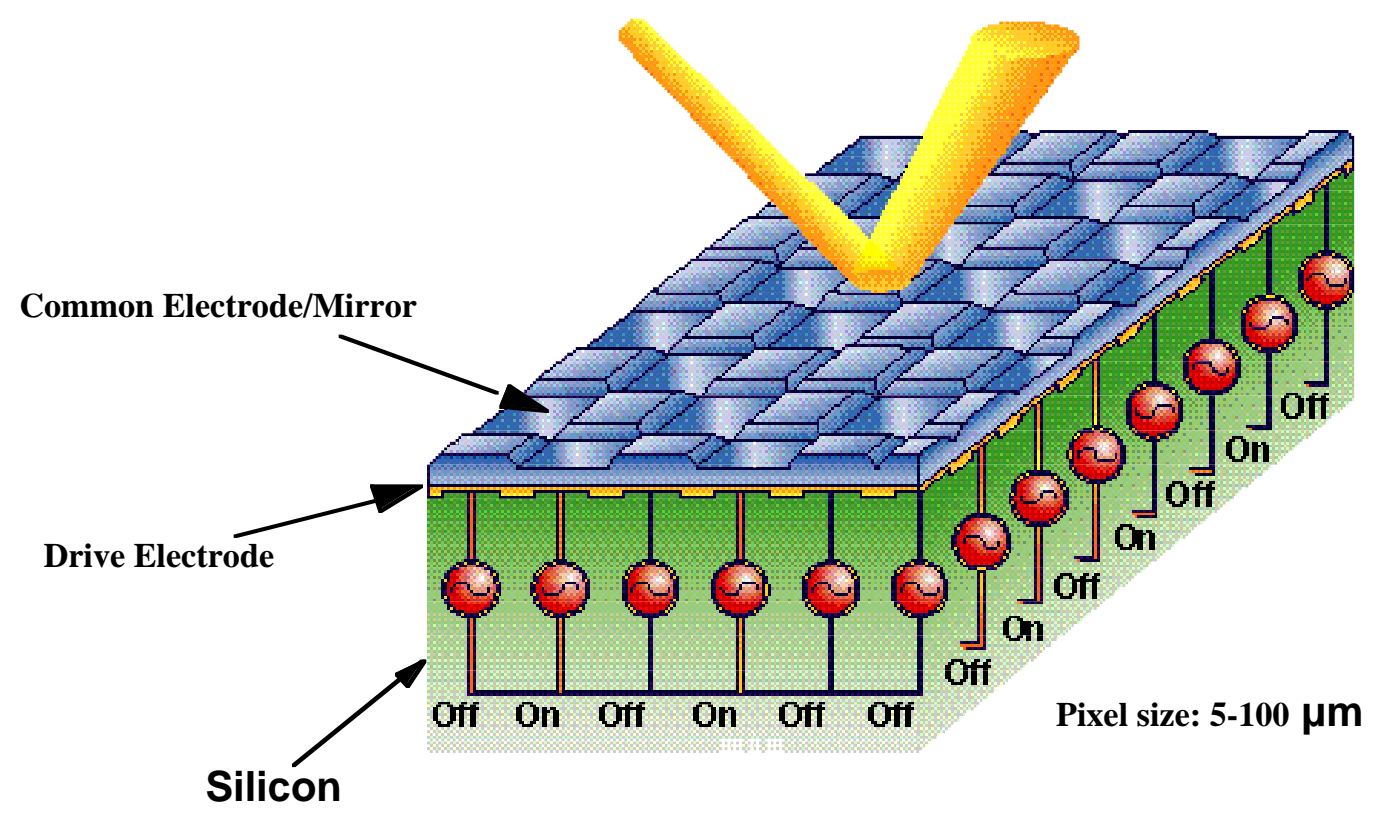


Figure 2. Voltage required to electrostatically compress various thin films $90 \mathrm{~nm}$ as a function of initial film thickness and Young's modulus.

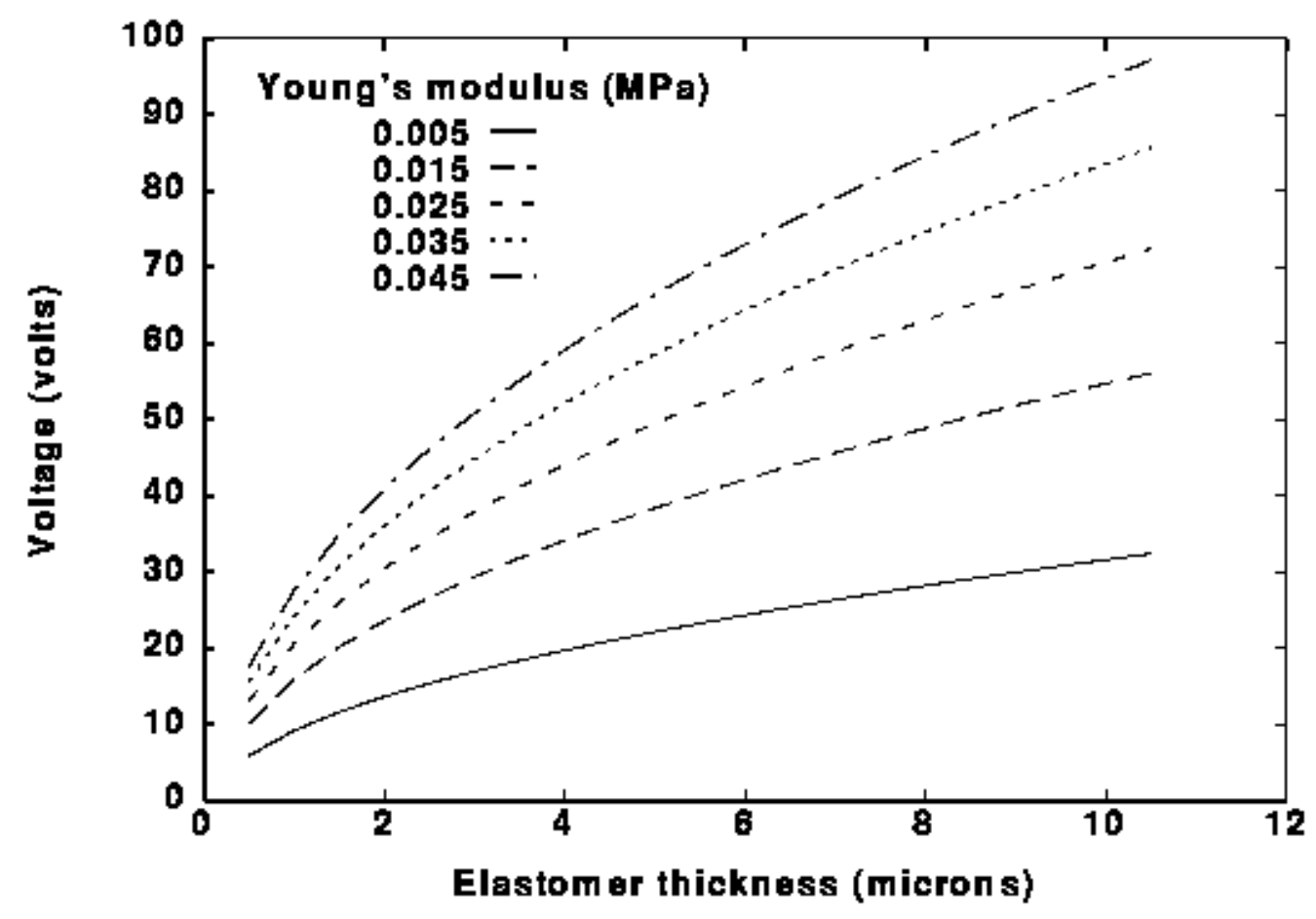

